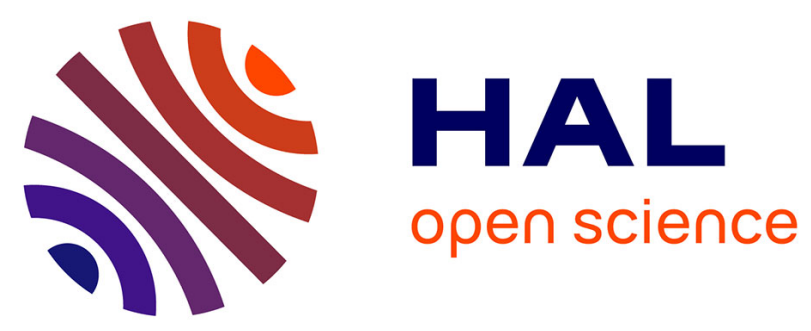

\title{
Simultaneous geometrical and material optimal design of hybrid elastomer/composite sandwich plates
}

\author{
Nicolas Le Maoût, Erwan Verron, Jerôme Bègue
}

\section{To cite this version:}

Nicolas Le Maoût, Erwan Verron, Jerôme Bègue. Simultaneous geometrical and material optimal design of hybrid elastomer/composite sandwich plates. Composite Structures, 2011, 93 (3), pp.11531157. 10.1016/j.compstruct.2010.10.008 . hal-01007306

\section{HAL Id: hal-01007306 https://hal.science/hal-01007306}

Submitted on 16 Jun 2014

HAL is a multi-disciplinary open access archive for the deposit and dissemination of scientific research documents, whether they are published or not. The documents may come from teaching and research institutions in France or abroad, or from public or private research centers.
L'archive ouverte pluridisciplinaire HAL, est destinée au dépôt et à la diffusion de documents scientifiques de niveau recherche, publiés ou non, émanant des établissements d'enseignement et de recherche français ou étrangers, des laboratoires publics ou privés. 


\title{
Simultaneous geometrical and material optimal design of hybrid elastomer/composite sandwich plates
}

\author{
N. Le Maoût ${ }^{a}, E$. Verron ${ }^{a}, J$. Bégué ${ }^{b}$ \\ a Institut de Recherche en Génie Civil et Mécanique, UMR CNRS 6183, Ecole Centrale de Nantes, BP 92101, 44321 Nantes cedex 3, France \\ ${ }^{\mathrm{b}}$ Centre Technique des Industries Mécaniques (CETIM), 74 route de la Jonelière, BP 82617, 44326 Nantes cedex 3, France
}

The paper presents the complete optimization of a hybrid elastomer/composite sandwich plate structure: design variables consist in the total number of layers of the structure, their respective thicknesses, their fiber orientations, the position(s) of the viscoelastic core(s) and the stacking sentence. The damping of the hybrid structure is calculated by the Method of Strain Energy (MSE). The constrained optimization maximizes the damping loss factor using the linear search algorithm. As an example, the method is applied to a simple structure and the results demonstrate the capabilities of our tool.
Keywords: Sandwich structures Passive damping Method of Strain Energy (MSE) Constrained optimization

\section{Introduction}

Nowadays, fiber-reinforced composites are widely used for automotive and aerospace parts because of their high stiffness, light weight, and also for their good fatigue and corrosion properties as compared to metals. Nevertheless, their damping properties are often revealed insufficient for some applications that involve vibrations or noise. In order to improve the damping properties of composites, the most common method is passive and consists in sticking an elastomeric viscoelastic patch (often combined with constrained metallic layer) in some well-chosen locations of the structure [1]. This solution is quite efficient but it increases both weight and cost of the structure. Moreover, as it is achieved a posteriori, both dimensions and mechanical response of the structure change as compared with those of the original structure.

An a priori solution consists in inserting interlaminar damping layer(s) in the composite material during the design of the structure $[2,3]$. Such a solution necessitates the determination of the more relevant position(s) and thickness(es) of damping layer(s) in the composite in order to maximize the modal loss factors without degrading the mechanical properties and increasing too much the total weight of the structure. In this case, the design of the hybrid structure must be achieved before manufacturing. Nevertheless, the definition of the best hybrid material is difficult because the dynamic properties of hybrid elastomer/composite depend on a large number of quantities such as the stacking sequence of the plies, the mechanical properties of the elastomer, ... [3-5]. To rationally overcome this difficulty, optimization procedures can be considered to determine the best dynamic properties in a given frequency range [6,7]. Until now, these studies have been limited to a small number of design variables such as thicknesses, ply angles or shear modulus of the elastomer layer(s).

The present paper proposes a complete optimization procedure in order to determine simultaneously the optimal number and locations of the elastomer and composite layers in the sandwich, in addition to the classical quantities mentioned above. This procedure is presented in the next section and its capabilities are illustrated in Section 3 and discussed in Section 4.

\section{An optimization tool for damped composites}

\subsection{Derivation of the problem}

Fig. 1 presents the problem. The sandwich plate studied here is rectangular and the design variables are:

- the number of layers $n$,

- the position of the viscoelastic layers $V_{i=1, \ldots, n}$,

- fiber orientations in the elastic layers $\theta_{i}=1, \ldots, n$ and,

- the thickness of each layer $t_{i=1, \ldots, n}$.

The aim of our optimization procedure is to maximize the damping capabilities of the material by inserting elastomeric viscoelastic layers at well-chosen positions, without degrading the mechanical properties and increasing too much the total mass of the structure. As examples, for 3 layers with 1 elastomeric layer there are 9 variables to optimize; for 5 layers with 2 elastomeric layers, 15 variables, and for 7 layers with 3 elastomeric layers, 21 variables. 


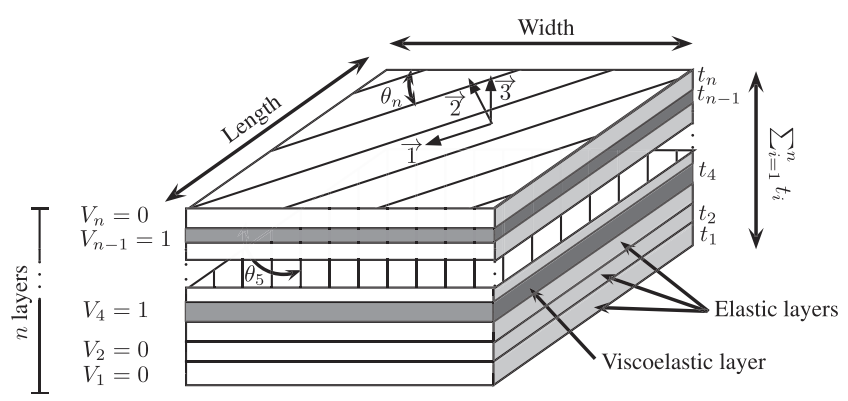

Fig. 1. The sandwich plate and the design variables.

\subsubsection{Geometry and materials}

For the geometry of the sandwich plate, the thickness of each layer is constrained to remain small as compared to both length and width of the plate in order to keep valid the plate assumption.

The fiber-reinforced layers are considered elastic, linear and orthotropic. Adopting the classical vectorial notation for both strain $\varepsilon$ and stress $\sigma$, and introducing the elastic compliance matrix, the constitutive equation reduces to [8]:

$$
\begin{aligned}
\left\{\begin{array}{l}
\varepsilon_{11} \\
\varepsilon_{22} \\
\varepsilon_{33} \\
\gamma_{12} \\
\gamma_{13} \\
\gamma_{23}
\end{array}\right\}= & {\left[\begin{array}{cccccc}
1 / E_{1} & -v_{21} / E_{2} & -v_{31} / E_{3} & 0 & 0 & 0 \\
-v_{12} / E_{1} & 1 / E_{2} & -v_{32} / E_{3} & 0 & 0 & 0 \\
-v_{13} / E_{1} & -v_{23} / E_{2} & 1 / E_{3} & 0 & 0 & 0 \\
0 & 0 & 0 & 1 / G_{12} & 0 & 0 \\
0 & 0 & 0 & 0 & 1 / G_{13} & 0 \\
0 & 0 & 0 & 0 & 0 & 1 / G_{23}
\end{array}\right] } \\
& \times\left\{\begin{array}{l}
\sigma_{11} \\
\sigma_{22} \\
\sigma_{33} \\
\sigma_{12} \\
\sigma_{13} \\
\sigma_{23}
\end{array}\right\}
\end{aligned}
$$

where $\varepsilon_{i j}$ and $\gamma_{i j}$ are the strain components, and $\sigma_{i j}$ are the stress components. The material parameters are the Young moduli $E_{i}$, the Poisson ratios $v_{i j}$ and the shear moduli $G_{i j}$. As shown in Fig. 1, the subscripts $(1,2,3)$ correspond to the fiber direction, the transverse direction and to the normal direction, respectively.

The rubber material used to damp the sandwich is isotropic, linear and viscoelastic. Moreover, the material properties are considered dependent on the loading frequency $f$. Introducing the fourth-order complex viscoelasticity tensor $\mathcal{D}^{\text {rubber }}$, the constitutive equation can be written as:

$\boldsymbol{\sigma}=\mathcal{D}^{\text {rubber }}(f) \boldsymbol{\varepsilon}$ with

$\mathcal{D}^{\text {rubber }}(f)=\mathcal{E}^{\Re}(f)+i \mathcal{E}^{\mathfrak{J}}(f)$

where $\mathcal{E}^{\Re}(f)$ and $\mathcal{E}^{\mathfrak{J}}(f)$ are the fourth-order tensors that characterize the energy storage and the dissipative response of the material, respectively. Similarly to Eq. (1), the use of the vectorial notation leads to the definition of the real compliance matrix

$\mathcal{E}^{\Re}=\Lambda\left[\begin{array}{cccccc}1-v(f) & v(f) & v(f) & 0 & 0 & 0 \\ v(f) & 1-v(f) & v(f) & 0 & 0 & 0 \\ v(f) & v(f) & 1-v(f) & 0 & 0 & 0 \\ 0 & 0 & 0 & \frac{1}{2}(1-2 v(f)) & 0 & 0 \\ 0 & 0 & 0 & 0 & \frac{1}{2}(1-2 v(f)) & 0 \\ 0 & 0 & 0 & 0 & 0 & \frac{1}{2}(1-2 v(f))\end{array}\right]$

and its complex counterpart

$\mathcal{E}^{\mathfrak{J}}=\Lambda^{*}\left[\begin{array}{cccccc}1-v(f)^{*} & v(f)^{*} & v(f)^{*} & 0 & 0 & 0 \\ v(f)^{*} & 1-v(f)^{*} & v(f)^{*} & 0 & 0 & 0 \\ v(f)^{*} & v(f)^{*} & 1-v(f)^{*} & 0 & 0 & 0 \\ 0 & 0 & 0 & \frac{1}{2}\left(1-2 v(f)^{*}\right) & 0 & 0 \\ 0 & 0 & 0 & 0 & \frac{1}{2}\left(1-2 v(f)^{*}\right) & 0 \\ 0 & 0 & 0 & 0 & 0 & \frac{1}{2}\left(1-2 v(f)^{*}\right)\end{array}\right]$

with $\Lambda=E(f) /(1+v(f)) /(1-2 v(f)), \quad \Lambda^{*}=\eta_{v}(f) E(f) /\left(1+v(f)^{*}\right) /\left(1-2 v(f)^{*}\right)$ and $v(f)^{*}=\eta_{v}(f) v(f)$. In this equation, $E(f)$ and $v(f)$ are the frequency-dependent Young modulus and Poisson ratio, and $\eta_{v}(f)$ is the loss factor of the elastomer.

\subsubsection{Loading conditions}

As mentioned in the introduction, the design of the damped structure is a compromise between the capability of dissipating energy and the maintenance of both the mass and the stiffness properties.

The dynamic response of the sandwich plate is determined by a classical free vibrations computation between 1 and $1000 \mathrm{~Hz}$, considering free-displacement boundary conditions on both $x$ - and $z$ edges (see Fig. 2 for the definition of the axes). By using the Method of Strain Energy (MSE in the following) [9], this calculation permits to determine the modal loss factors $\eta_{i}$ of the structure for each modal frequency $i$ in the range $[1 \mathrm{~Hz}, 1000 \mathrm{~Hz}]$. The $i^{\text {est }}$ modal loss factor for which the natural frequency is equal to $f_{i}$ is given by

$\eta_{i}=\eta_{v}\left(f_{i}\right) \frac{W}{W_{\text {tot }}}$

where $W$ is the strain energy of the viscoelastic core and $W_{\text {tot }}$ is the total strain energy of the structure. It is to note that as the elastomer properties explicitly depend on the loading frequency, this calculation is iterative for each eigenfrequency.

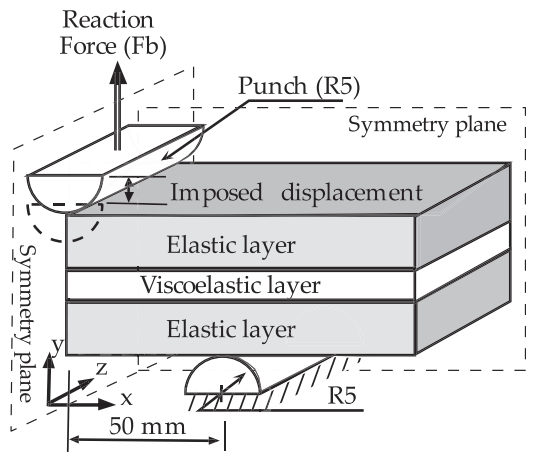

(b)

Fig. 2. (a) Tensile tests and (b) three-point bending test for a three-layers sandwich plate with symmetry conditions. 
Moreover, the new structure must withstand static loading conditions, i.e. preserves a sufficient stiffness. Here, we choose two different static loading conditions: tensile and bending conditions. First, we study extension stiffness properties by considering uniaxial extension in the two planar directions as shown in Fig. 2a. Two simulations are performed under uniaxial prescribed displacement in the $x$ and $z$-directions, and in both cases the reaction forces Ftx and $F t z$ are calculated. Second, we consider the bending stiffness of the structure. This is achieved by a three-point bending calculation as shown in Fig. 2b: a central punch imposes a few millimeters of deflection in the center of the laminated structure and the reaction force at the center $\mathrm{Fb}$ is calculated.

\subsubsection{Optimization}

The optimization problem can now be established. The objective function $\mathcal{F}$ to be minimized is simply the reciprocal average of the loss factors:

$\left.\mathcal{F}=\frac{1}{N} \sum_{j=1}^{N} \eta_{j}\right)^{-1}$

in which $N$ is the number of eigenfrequencies in the range [1 $\mathrm{Hz}, 1000 \mathrm{~Hz}$.

The minimization of $\mathcal{F}$ is constrained by the mass increase and stiffness decrease requirements. Before the optimization, the values of reference for the initial (undamped, i.e. without viscoelastic layers) structure are calculated: its mass $M_{r}$, and the static reaction forces $F t x_{r}, F t z_{r}$ and $F b_{r}$ which are the undamped counterparts of the previously defined forces Ftx, Ftz and $F b$ (the subscript ${ }_{{ }_{r}}$ stands for reference structure). The non-linear constraints $\left(C_{n l}, i\right)_{i=1,4}$ consist in limiting the change in both mass and stiffness properties:

$$
\begin{aligned}
& C_{n l} 1: \frac{M_{r}-M}{M_{r}}-\mathrm{Tol}_{M} \leqslant 0 \\
& C_{n l} 2: \frac{F b_{r}-F b}{F b_{r}}-\mathrm{Tol}_{F b} \leqslant 0 \\
& C_{n l} 3: \frac{F t x_{r}-F t x}{F t x_{r}}-\mathrm{Tol}_{F t x} \leqslant 0 \\
& C_{n l} 4: \frac{F t z_{r}-F t z}{F t z_{r}}-\mathrm{Tol}_{F t z} \leqslant 0
\end{aligned}
$$

where $\mathrm{Tol}_{M}, \mathrm{Tol}_{F b}$, $\mathrm{Tol}_{F t x}$ and $\mathrm{Tol}_{F t z}$ are the user-defined tolerances on each quantity. Moreover, we add a linear constraint $C_{l} 1$ to limit the total stiffness of the damped sandwich to MaxThickness:

$C_{l} 1: \sum_{i=1}^{n} t_{i} \leqslant$ MaxThickness

\subsection{Implementation}

\subsubsection{Finite element computations}

It is well-known that the dissipation is mainly due to transverse shearing of the viscoelastic layers (see for example [10]). In order to predict shearing and to improve the bending response of the numerical model, 3D linear brick elements with incompatible modes are considered for the rubber layers. Moreover, the elastic layers are also meshed with this type of elements because it highly simplifies the optimization of the rubber layers: in the optimization loop, the material properties of the layers can be changed without remeshing. The finite element model is presented in Fig. 3. For the three-point bending calculations, tools are modeled as analytical tools and contact with friction $(\mu=0.12)$ is considered. Finally, the eigenfrequency problem of the undamped structure is solved with the help of the Lanczos algorithm.

As the finite element model uses only brick elements, the number of degrees of freedom is large and it implies significant CPU

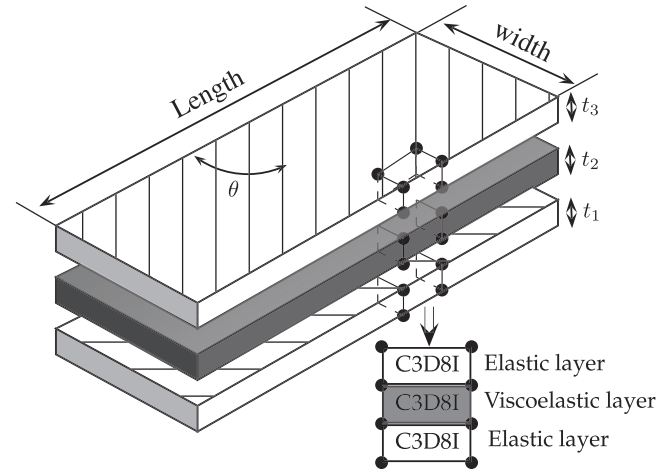

Fig. 3. Several design variables on FEM modelling with the incompatible mode elements.

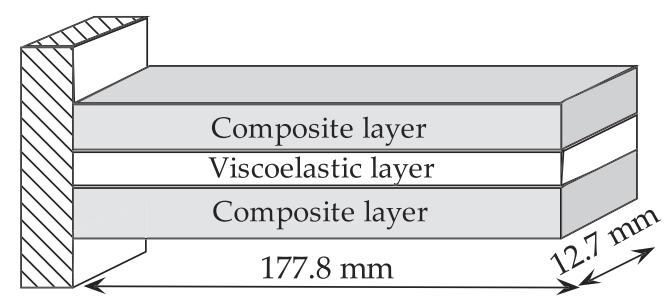

Fig. 4. A three-layer cantilever sandwich beam used to determine the optimal mesh size.

time. In order to limit CPU time, we preliminarily determine an optimal mesh size by considering a three-layer cantilever beam with isotropic materials as shown in Fig. 4; this structure has been chosen because it was thoroughly studied theoretically, numerically and experimentally [11-13]. The in-plane optimal mesh size is $0.55 \mathrm{~mm}$ as compared to the size of the structure (see Fig. 4). Moreover, we proved that a single element in the thickness direction of the viscous layer is sufficient to capture the phenomena, if the thickness is smaller than $1 \mathrm{~mm}$.

\subsubsection{The optimization algorithm}

The minimization of the average loss factor Eq. (7) by the MSE with the constraints Eq. (8) is performed by the active-set algorithm and the Hessian of the problem is computed by a quasiNewton method.

\subsubsection{The tool}

The global problem of optimization is solved by coupling Matlab and Abaqus:

- Matlab is used to find the minimum of the constrained nonlinear multi-variable function,

- Abaqus is used to solve the static problems,

- Matlab and Abaqus are conjointly used to determine iteratively the damped frequencies by updating the viscoelastic properties,

- Finally, Python scripts link both softwares.

The flow of the numerical tool is summarized in Fig. 5.

\section{Results}

In order to demonstrate the capabilities of our methodology, we study the optimization of a 5-layer rectangular sandwich plate. Its in-plane dimensions are $200 \mathrm{~mm} \times 125 \mathrm{~mm}$; the stacking sequence is [0/45/0/45/90] and the thickness of the layers is equal to $0.2 \mathrm{~mm}$ for the four first layers and $0.4 \mathrm{~mm}$ for the last one. The material is a glass fiber-reinforced epoxy composite and the 


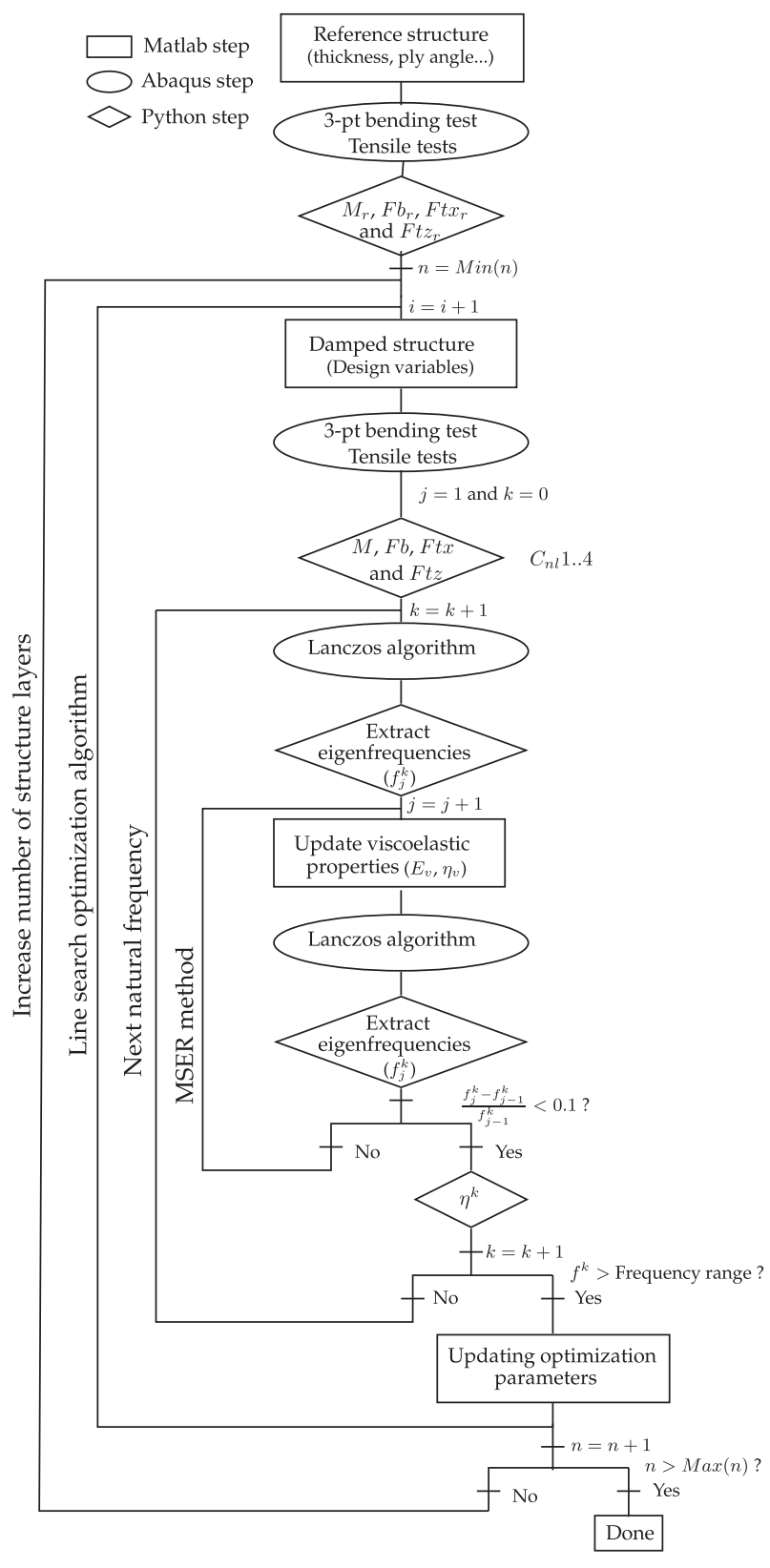

Fig. 5. Flow of the numerical tool.

Table 1

Bounds applied to the geometrical parameters.

\begin{tabular}{lll}
\hline Design variables & Lower bound & Upper bound \\
\hline Nb of layers $n$ & 4 & 7 \\
Layer thickness $t$ & $0.1 \mathrm{~mm}$ & $3 \mathrm{~mm}$ \\
Layer orientation $\theta$ & $0^{\circ}$ & $90^{\circ}$ \\
\hline
\end{tabular}

following mechanical properties, issued from [14], are considered: $E_{1}=29.9 \mathrm{GPa}, E_{2}=E_{3}=7.5 \mathrm{GPa}, G_{12}=2.25 \mathrm{GPa}$, and $v_{12}=v_{23}=v_{12}=$ 0.24 . Moreover, its density is equal to $1500 \mathrm{~kg} \mathrm{~m}^{-3}$. The problem consists in damping this structure with an elastomer. The material parameters are those of [4]: the Young modulus is frequencydependent

$E(f)=0.0041+0.0322 \log (f) \quad$ GPa,

and the loss factor is constant and equal to $\eta_{v}=0.3$. The Poisson ratio is also constant and equal to $v=0.3$; and the density is $\rho=968.1 \mathrm{~kg} \mathrm{~m}^{-3}$. The constraints adopted for the optimization are as follows: the possible loss in stiffness and increase in mass between the new structure and the reference one are set to $5 \%$, the maximum total thickness is limited to $5 \mathrm{~mm}$ and the geometrical parameters are bounded by the values given in Table 1 . Initial values of both thicknesses and layer orientations are randomly chosen.

Finally, all the calculations have been made on a cluster with $6 \times$ Quad-Core AMD Opteron(tm) Processor 8380, $2500 \mathrm{MHz}$ processor with $65 \mathrm{~Gb}$ RAM and $\mathrm{CPU}$ used $=6$.

The optimization of the structure is performed for $n=3$ to 7 layers. The results and their comparison with the reference structure data are presented in Table 2. First, results with 4 and 6 layers are not shown because they are identical to those with 3 and 5 layers respectively, i.e. the same number of viscoelastic layers and two successive composite layers with the same ply angle. In every cases, ply angles are $0^{\circ}$ or $90^{\circ}$. Moreover, the comparison of the mass and stiffness of damped plates with those of the reference plate shows that all constraints (linear and non-linear) are respected.

Fig. 6 presents the modal loss factors for each optimized damped structure for each eigenfrequency. As eigenmodes alternate from bending to torsion, one can observe an abrupt change in the modal loss factor between two successive eigenfrequencies. Even if results present a similar trend for 3, 5 and 7-layer plates, the best structure is the 7-layer one that contains 3 viscoelastic layers and which stacking sequence is $[0 / \mathrm{V} / 90 / \mathrm{V} / 0 / \mathrm{V} / 90]$.

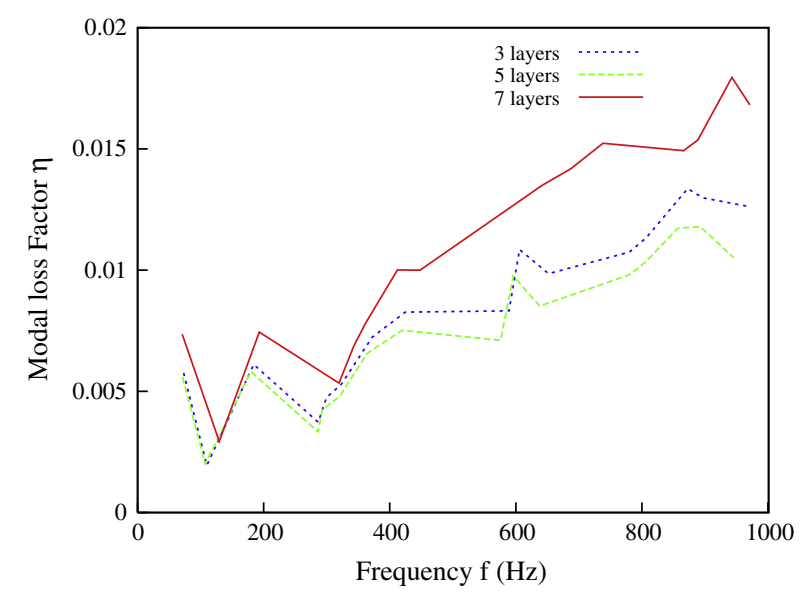

Fig. 6. Optimized modal loss factors for a sandwich plate with $\eta_{v}=0.3$.

Table 2

Design parameters and constraints after optimization on the sandwich plate for $\eta_{v}=0.3$ (' $\mathrm{V}$ ' denote the position of the viscoelastic layer).

\begin{tabular}{lllll}
\hline & Reference & 3 layers & 5 layers & 7 layers \\
\hline$\theta\left({ }^{\circ}\right)$ & {$[0 / 45 / 0 / 45 / 90]$} & {$[0 / \mathrm{V} / 90]$} & {$[0 / \mathrm{V} / 90 / \mathrm{V} / 90]$} & {$[0 / \mathrm{V} / 90 / \mathrm{V} / 0 / \mathrm{V} / 90]$} \\
$t(\mathrm{~mm})$ & {$[0.2 / 0.2 / 0.2 / 0.2 / 0.4]$} & {$[0.57 / 0.34 / 0.47]$} & {$[0.59 / 0.22 / 0.18 / 0.1 / 0.29]$} & {$[0.25 / 0.14 / 0.27 / 0.22 / 0.22 / 0.1 / 0.23]$} \\
$M(\mathrm{~kg})$ & 0.045 & $0.0472(+4.95 \%)$ & $0.0473(+5 \%)$ & $0.0473(+5 \%)$ \\
$F b(\mathrm{~N})$ & -25.25 & $-24(-5 \%)$ & $-24.36(-3.5 \%)$ & $-23.99(-5 \%)$ \\
$F t x(\mathrm{kN})$ & -21.46 & $-20.46(-4.7 \%)$ & $-20.62(-3.9 \%)$ & $-20.39(-5 \%)$ \\
$F t z(\mathrm{kN})$ & 13.10 & $14.38(+10 \%)$ & $14.81(+13 \%)$ & $12.45(-5 \%)$ \\
& Nb. iterations & 46 & 175 & 364 \\
& CPU time (h) & 18 & 98 & 227 \\
\hline
\end{tabular}




\section{Discussion}

First, we can remark that the optimized orientations $\theta_{i}$ of the elastic layers differ from those of the original structure $\left(45^{\circ}\right)$ and are only equal to $0^{\circ}$ and $90^{\circ}$. It is due to the choice of the static stiffness tests in the optimization process: as only two directions are considered for the tensile and bending tests, the stiffness requirements of the constraints lead to these orientations. Nevertheless, specifying several stiffness experiments in different directions in the optimization process would lead to other orientations.

Second, the active-set algorithm generally converges and is well-recognized for its ability to solve large dimension problems. However, it has the disadvantage to converge to local minima, especially when the optimization problem involves numerous variables. This is the case here; as an example, the result obtained with five layers admits worse damping capabilities than the result obtained with the 3-layer structure. In fact, considering different number of layers prevents from using a global search of the solution because of the significant increase in computation time it would lead to; these time consuming methods being incompatible with industrial applications. Indeed, considering different optimization algorithms is required.

Finally, we observe that the damping efficiency is always weaker for low eigenfrequencies than for high ones. It can be easily explained by recalling that the damping capabilities of the structure are defined by the ratio of the damped energy with respect to the total energy of the structure. Indeed, the total strain energy of the whole structure is mainly driven by the energy in the composite layers that do not change a lot. The strain energy of the elastomeric layers depends on the Young modulus $E(f)$ that increases with the frequency (see Eq. (10)), and as the rubber loss factor $\eta_{v}$ is set constant in the example, the strain energy of the elastomeric layers increases with the frequency. In fact, $\eta_{v}$ also depends on the frequency, more precisely it increases as $f$ increases; so this dependency would compensate for the increase of the damping efficiency. Taking into account the increase of $\eta_{v}$ with $f$ does not lead to theoretical or numerical difficulties, but it necessitates more complex experiments to characterize the material response.

\section{Conclusion}

In this paper, a numerical tool developed to maximize the passive damping capabilities of multi-layer plates with respect to a large number of variables has been presented. Optimization is per- formed with linear and non-linear constraints to limit the total mass and to ensure a minimum stiffness of the hybrid structure. The problem is solved using the optimization line-search algorithm in Matlab, coupled with the FEA software Abaqus. Passive damping properties of the structure, including frequency-dependent viscoelastic core, is estimated using the Method of Strain Energy.

To illustrate the approach, we have optimized a simple structure composed of layers of composite and viscoelastic rubber. The results consist in hybrid materials that maximize the passive damping properties of the structure with respect to both design constraints and bounds on parameters. With this example, we have demonstrated the efficiency of our tool to optimize very different types of parameters, considering the frequency-dependent properties of the elastomer core.

\section{References}

[1] Zheng H, Cai C, Tan XM. Optimization of partial constrained layer damping treatment for vibrational energy minimization of vibrating beams. Comput Struct 2004;82:2493-507.

[2] Kishi H, Kuwata M, Matsuda S, Toshihiko A, Atsushi M. Damping properties of thermoplastic-elastomer interleaved carbon fiber-reinforced epoxy composites. Compos Sci Technol 2004;64:2517-23.

[3] Rao M, Echempati R, Nadella S. Dynamic analysis and damping of composite structures embedded with viscoelastic layers. Composites Part B 1997;28: 547-54.

[4] Zhang SH, Chen HL. A study on the damping characteristics of laminated composites with integral viscoelastic layers. Compos Struct 2006;74:63-9.

[5] Meunier M, Shenoi RA. Dynamic analysis of composite sandwich plates with damping modelled using high-order shear deformation theory. Compos Struct 2001;54:243-54.

[6] Baz A, Ro J. Optimum design and control of active constrained layer damping. J Vib Acoust 1995;117(B):135-45.

[7] Araùjo AL, Mota Soares CM, Mota Soares CA, Herskovits J. Optimal design and parameter estimation of frequency dependent viscoelastic laminated sandwich composite plates. Compos Struct 2010;92(9):2321-7.

[8] Reddy JN. Mechanics of laminated composite plates and shells - Theory and analysis. 2nd ed. Boca Raton, Florida: CRC Press; 2004.

[9] Ungar EE, Kerwin Jr EM. Loss factors of viscoelastic systems in terms of strain energy. J Acoust Soc Am 1962;34(2):954-62.

[10] Kerwin EM. Damping of flexural waves by a constrained viscoelastic layer. J Acoust Soc Am 1959;37(7):952-62.

[11] Soni ML. Finite element analysis of viscoelastically damped sandwich structures. Shock Vib Bull 1981;55(1):97-109.

[12] Daya EM, Potier-Ferry M. A numerical method for nonlinear eigenvalue problems application to vibrations of viscoelastic structures. Comput Struct 2001;79:533-41.

[13] Johnson CD, Kienholz DA, Rogers LC. Finite element prediction of damping in beams with constrained viscoelastic layers. Shock Vib Bull 1981;51(1): 71-81.

[14] Berthelot J-M, Assarar M, Sefrani Y, El Mahi A. Damping analysis of composite materials and structures. Compos Struct 2008;85:189-204. 